\title{
Water Deficit and Recovery-Induced Changes in Growth, Photosynthetic Characteristics, Antioxidant Enzymes and Yield of Two Tartary Buckwheat Genotypes
}

\author{
Yan Wan ${ }^{1,2}$, Jianyong Ouyang ${ }^{1,2}$, Xuxiao Gong ${ }^{1,2}$, Liqing $\mathrm{Le}^{1,2}$, Xiaoyong $\mathrm{Wu}^{1,2}$, Qi $\mathrm{Wu}^{1,2}$, Liang $\mathrm{Zou}^{1,2}$, Gang $\mathrm{Zhao}^{1,2}$ \\ and Dabing Xiang ${ }^{1,2^{*}}$ \\ ${ }^{1}$ Key Laboratory of Coarse Cereal Processing, Ministry of Agriculture and Rural Affairs, Chengdu University, Chengdu \\ 610106, Sichuan, China \\ ${ }^{2}$ School of Food and Biological Engineering, Chengdu University, Chengdu 610106, Sichuan, China \\ "For correspondence: dabing.xiang@163.com; yanwan@cdu.edu.cn \\ Received 21 October 2020; Accepted 17 November 2020; Published 10 January 2021
}

\begin{abstract}
Tartary buckwheat (Fagopyrum tataricum) is an important food crop that is widely adaptable to hostile environments. In this study the responses of two Tartary buckwheat genotypes: drought-susceptible Chuanqiao No. 1 (CQ) and drought-tolerant Jingqiao No. 2 (JQ) in terms of morphology, photosynthesis, physiology and yield to a progressive water deficit and recovery treatment (WD-R) were evaluated. Plants in the well-watered (WW) treatment were watered throughout the experiment. Compared to the WW treatment, water deficit in the WD-R treatment caused decreases in plant height, stem diameter, branch number, stem node number, biomass, seed number, soil water content (SWC), leaf relative water content (RWC), net photosynthesis rate $(\mathrm{Pn})$, intercellular $\mathrm{CO}_{2}$ concentration, stomatal conductance (Gs), transpiration rate ( $\mathrm{Tr}$ ) and Fv/Fm in both $\mathrm{CQ}$ and JQ plants. Leaf wilting, malondialdehyde content, superoxide dismutase activity, peroxidase activity, initial fluorescence $\left(\mathrm{F}_{0}\right)$ and root-to-shoot ratio were significantly increased under water stress in the WD-R treatment. Under the WD-R treatment, compared to CQ, JQ maintained higher RWC, SWC, Pn, Gs, WUE, Fv/Fm, plant height, branch number, stem node number, root biomass, stem biomass, leaf biomass, total biomass, root-to-shoot ratio, seed number per plant, and yield, but a lower $\operatorname{Tr}$ and $\mathrm{F}_{0}$. By correlation analysis, Gs was positively correlated with leaf RWC and SWC. These differential growth indexes, biochemical traits and physiological responses might be useful for understanding drought-tolerance genotypes that can grow under water-deficit conditions with minimum yield loss. (C) 2021 Friends Science Publishers
\end{abstract}

Keywords: Water deficit and recovery; Photosynthetic parameter; WUE; Antioxidant enzyme; Yield loss

\section{Introduction}

Water deficit is a primary constraint for terrestrial plants (Chimungu et al. 2014) and drought stress greatly limits crop production and food stability in arid and semiarid regions (Delpérée et al. 2003; Farooq et al. 2009). Plants have evolved various strategies to deal with drought stress, which can be divided into drought avoidance, and the development of adaptation mechanisms that contribute to drought tolerance (Claeys and Inze 2013; Hussain et al. 2018). Adaptive strategies that are induced during the drought process help plants survive (Nguyen et al. 2016). Plants such as maize (Zea mays L.) reduce tissue dehydration, either via tolerance to lower tissue water potential or by maintaining the water potential $(\mathrm{Wu}$ and Cosgrove 2000; Farooq et al. 2017, 2018). To successfully minimize water loss, plants limit transpiration by decreasing leaf area, closing their stomata and accelerating leaf senescence, resulting in improved water-use efficiency (WUE) (Franks et al. 2015; Farooq et al. 2019) or reduced photosynthesis (Shi et al. 2016). To protect against droughtinduced oxidative damage to the cells, osmotically active metabolites rapidly increase (Vasquez-Robinet et al. 2008; Claeys and Inze 2013). Plants also respond to water deficit by inducing sets of both regulatory and functional genes (Yoshida et al. 2014; Simmons and Bergmann 2016). Recent studies in Arabidopsis (Arabidopsis thaliana) have shown that phytohormones such as cytokinin or abscisic acid induce signaling pathways that activate or negatively regulate the genes necessary for drought-acclimation responses (Yoshida et al. 2014; Nguyen et al. 2016). Transcriptional regulation is a vital regulatory mechanism mediating drought tolerance (Yoo et al. 2010; Simmons and Bergmann 2016).

Tartary buckwheat (Fagopyrum tataricum) is an important traditional edible and medicinal crop with 
excellent nutritional and pharmacological properties (Zhu 2016). Tartary buckwheat is usually cultivated in the mountainous areas of western China, northern India, Bhutan, and Nepal at high altitude. Therefore, it is extremely well-adapted to harsh climatic conditions (Zhou et al. 2015; Zhang et al. 2017) and it regularly suffers from drought stress in its main growing regions (Xiang et al. 2020). Thus, Tartary buckwheat is an ideal plant to study adaptation mechanisms under drought conditions. Therefore, to better understand the specific drought-adaptive responses with respect to morphological plasticity, biochemical indexes, photosynthetic and chlorophyll fluorescence parameters, WUE and seed yield, two contrasting Tartary buckwheat genotypes [droughtsusceptible Chuanqiao No. 1 (CQ) and drought-tolerant Jinqiao No. 2 (JQ)] were sown under progressive drought and recovery at flowering stage.

\section{Materials and Methods}

\section{Growth conditions and materials}

The experiment was conducted in a greenhouse at Chengdu University $\left(30^{\circ} 39^{\prime} \mathrm{N}, 104^{\circ} 11^{\prime} \mathrm{E}\right.$, altitude $490 \mathrm{~m}$ asl). Two Tartary buckwheat genotypes, previously evaluated for their yield responses to water stress, were used in this experiment: the drought-tolerant cv. JingQiao No. 2 (JQ) and drought-susceptible cv. ChuanQiao No. 1 (CQ), selected for by the Shanxi Academy of Agricultural Sciences and the Agricultural Science Institute of Zhaojue County, Liangshan Yi Autonomous Prefecture, respectively. The experiment was initiated on 4 Mar 2018. Seeds were sterilized with $0.1 \%$ potassium permanganate solution for $30 \mathrm{~min}$ and then rinsed three to four times with distilled water. They were then sown in plastic pots $(20 \mathrm{~cm}$ high, 25 $\mathrm{cm}$ diameter) in a waterproof shelter. One plant was allowed to grow per plastic pot to the three-and-a-half leaf stage. The plastic pots were allowed to drain freely from the bottom and contained $15 \mathrm{~kg}$ soil (sandy loam, with $1.87 \mathrm{~g} \mathrm{~kg}^{-1}$ total nitrogen, $17.3 \mathrm{~g} \mathrm{~kg}^{-1}$ organic matter, $1.49 \mathrm{~g} \mathrm{~kg}^{-1} \mathrm{P}_{2} \mathrm{O}_{5}$, and $15.8 \mathrm{~g} \mathrm{~kg}^{-1}$ total potassium). At the experimental site of Chengdu University, the annual mean air temperature was $16^{\circ} \mathrm{C}$, total precipitation was $900-1300 \mathrm{~mm}$, and average sunshine duration was $1042-1412$ h overall year.

\section{Drought treatment and recovery}

The pots were weighed daily. A fully watered pot was set at $100 \%$ water-holding capacity. For the well-watered (WW) treatment, plants were watered daily to maintain $90 \%$ water-holding capacity. For the water deficit and recovery (WD-R) treatment, plants from bud-appearing stage were exposed to gradual soil water depletion $(50 \%$ water-holding capacity) for 14 days, then all plants were re-watered daily to the initial pot water-holding capacity for 9 days of recovery. Pots were weighed daily during both water-deficit and recovery periods. There were 60 pots for each treatment. Each treatment had four independent replicates, arranged in a completely randomized designed. The imposition of drought simulated possible conditions in the field.

\section{Microenvironment for plants}

During the experiment, soil water content (SWC) and soil electrical conductivity (SEC) were measured in the top $20 \mathrm{~cm}$ of the soil every 2 days using a WET-2-K1 time-domain reflectometry probe (Delta-T Devices Ltd., Cambridge, U.K.).

\section{Evaluation of leaf wilting ( $\mathrm{LW})$ and leaf relative water content (RWC)}

To analyze LW, 10 plants from 10 pots of each treatment were monitored daily at 08:00 h. LW was graded on a fivepoint scale: level 1, normal; level 2, curling slightly; level 3, curling slightly with middle and lower leaves drooping; level 4, curling heavily with all leaves drooping; level 5, rolling into a cylindrical shape with all leaves drooping and the tip growth point wilting.

To measure leaf RWC, leaf samples were weighed for fresh weight $(\mathrm{FW})$ and drenched with deionized water at $4^{\circ} \mathrm{C}$ for saturated weight (SW), then oven-dried at $80^{\circ} \mathrm{C}$ for $72 \mathrm{~h}$ for dry weight (DW). Leaf RWC (\%) was calculated as:

$$
\left[\frac{\text { FW-DW }}{\mathrm{SW}-\mathrm{DW}}\right] \times 100
$$

(Barrs and Weatherley 1962)

\section{Gas exchange traits}

The net photosynthesis rate $(\mathrm{Pn})$, intercellular $\mathrm{CO}_{2}$ concentration (Ci), stomatal conductance (Gs) and transpiration rate (Tr) of the last fully developed and expanded leaf was examined on days 5, 9, 14, 19 and 23 of the experimental period with a portable photosynthesis system (LI-6400, LI-COR Biosciences, Lincoln, NE, USA). These parameters were automatically recorded by the machine at 10:00-12:00 h under atmospheric $\mathrm{CO}_{2}$ and full sunlight. WUE was calculated as:

\section{$\mathrm{Pn} / \mathrm{Tr}$}

\section{Chlorophyll (Chl) $\boldsymbol{a}$ fluorescence}

Chl $a$ fluorescence of the same leaves used for the gasexchange measurements was determined in leaf discs by pulse-amplitude modulated fluorescence spectrometry (Mini-PAM, Heinz Walz, Pfullingen, Germany). The initial Chl $a$ fluorescence $\left(\mathrm{F}_{0}\right)$ was recorded after 30 min dark adaptation using a beam of $0.2 \mu \mathrm{mol} \mathrm{m} \mathrm{m}^{-1} \mathrm{~s}^{-1}$. The maximum Chl $a$ fluorescence (Fm) was recorded at $8,000 \mu \mathrm{mol} \mathrm{m}^{-1} \mathrm{~s}^{-1}$ with a 0.8 -s saturating pulse. The Overall Chl $a$ fluorescence 
(Fv) was calculated as $\mathrm{Fm}-\mathrm{F}_{0}$. Fv/Fm, the maximum efficiency of photosystem II (PSII) photochemistry in the dark, was calculated as:

$$
(\mathrm{Fm}-\mathrm{Fo}) / \mathrm{Fm}
$$

(Dias and Bruggemann 2010)

\begin{abstract}
Malondialdehyde (MDA) and soluble protein (SP) contents, and activities of superoxide dismutase (SOD) and peroxidase (POD)
\end{abstract}

Fresh leaf samples (last fully developed leaves) were taken on the same days as the photosynthesis measurements to determine MDA and SP contents, and the activities of SOD and POD. Leaf samples were immediately frozen in liquid nitrogen after collection and then stored at $-80^{\circ} \mathrm{C}$ for further use. Contents of MDA and SP were measured with suitable modifications of Dhindsa's method (Dhindsa et al. 1981) and Bradford's method (Bradford 1976), respectively. The activities of SOD and POD were determined by Giannopolitis and Ries' method (Giannopolitis and Ries 1977) and Nakano and Asoda's method (Nakono and Asada 1980), respectively.

\section{Morphological measurements and grain yield}

In each treatment, 20 plants from 20 pots of each treatment were harvested at physiological maturity to determine plant height, branch number, stem diameter, and number of nodes on the main stem. Seed numbers for each plant, 1000-seed weight and grain yield were recorded.

\section{Statistical analysis}

Statistical analyses were carried out using Excel 2010, S.P.S.S. 13.0 (Chicago, I.L., U.S.A.) and SigmaPlot 10.0 (Aspire Software International, Ashburn, V.A., U.S.A.). Two-way ANOVA was used to determine the significance level, and means were compared by Duncan's multiple range tests at a significance level of $P<0.05$.

\section{Results}

\section{Leaf RWC and LW}

During the experiment, no significant fluctuations in leaf RWC (Fig. 1A) or LW (Fig. 1B) were observed in either CQ or JQ plants under the WW treatment. A gradual decrease in RWC for both Tartary buckwheat cultivars were observed during the drought part of the WD-R treatment, but the JQ plants maintained a higher RWC level than the CQ plants. On day 14 of the WD-R treatment, the RWC of CQ leaves was $48.2 \%$ and that of JQ was $72.5 \%$. Upon rewatering, RWC increased in both cultivars. When subjected to water deficit, LW markedly increased for the two genotypes, but was significantly lower for JQ plants vs. CQ plants. On day 14 of the WD-R treatment, CQ leaves showed a high level of drooping and tip growth point wilting, whereas JQ leaves were slightly curled with middle and lower leaf drooping. After re-watering, the LW in both genotypes gradually returned to the WW levels.

\section{Soil water content (SWC) and soil electrical conductivity (SEC)}

As shown in Fig. 2A and B, neither SEC nor SWC changed significantly in the CQ or JQ pots under the WW treatment. SEC in the WD-R pots of both genotypes increased significantly during the drought part of the treatment compared to the WW treatment. On day 14 of the WD-R treatment, SEC in the CQ pots was significantly higher than that in the JQ pots. After re-watering, SEC in the WD-R pots of both genotypes gradually decreased to normal WW values. Opposite trends were observed for SWC: SWC in $\mathrm{CQ}$ and JQ pots decreased by $44.6-51.1 \%$ during the dehydration part of the WD-R treatment compared to the WW treatment. On day 14 of the WD-R treatment, SWC in CQ pots had decreased more than in the JQ pots. During the process of recovery in the WD-R treatment, the SWC in CQ and JQ pots increased back to WW values.

\section{Temporal dynamics of photosynthetic characteristics and WUE}

All measured photosynthetic parameters and WUE of CQ and JQ plants in the WW treatment remained stable during the experiment (Fig. 3A-D). Pn, Ci, Gs and $\mathrm{Tr}$ of both genotypes declined consistently with decreasing soil water availability during drought phase of the WD-R treatment. Pn, $\mathrm{Gs}, \mathrm{Ci}$ and $\mathrm{Tr}$ progressively increased with water application during the recovery phase of the WD-R treatment. There were significant differences in Pn and Gs between the two genotypes: JQ plants showed higher Pn than CQ plants during the experiment. Pn, Gs, and Tr of the CQ and JQ plants differed significantly $(P<0.05)$ between WW and WD-R treatments on days 5, 9, 14, 19 and 23, while $\mathrm{Ci}$ differed on days 5, 9, 14 and 19. On day 14, Pn and Ci in CQ plants under the WD-R treatment were lowest among all treatments and genotypes, reduced by 81.08 and $56.44 \%$, respectively, compared to JQ plants in the WW treatment $(P$ $<0.01)$. Gs and Tr in JQ plants under WD-R treatment were significantly lower than in the other treatments. There was no difference in WUE between the two genotypes in the WW treatment (Fig. 4). WUE in the JQ plants under the WD-R treatment was significantly highest for all treatments and genotypes on days 5, 9, 14 and 19. Especially on day 14, WUE in JQ plants under WD-R treatment was at its highest point in the whole experimental phase.

\section{Dynamic changes in $\mathrm{Chl}$ a fluorescence characteristic}

$\mathrm{F}_{0}$ and $\mathrm{Fv} / \mathrm{Fm}$ of $\mathrm{CQ}$ and JQ plants in the WW treatment were relatively constant throughout the experiment (Fig. 5A and $\mathrm{B}$ ). However, $\mathrm{F}_{0}$ was significantly increased by water 


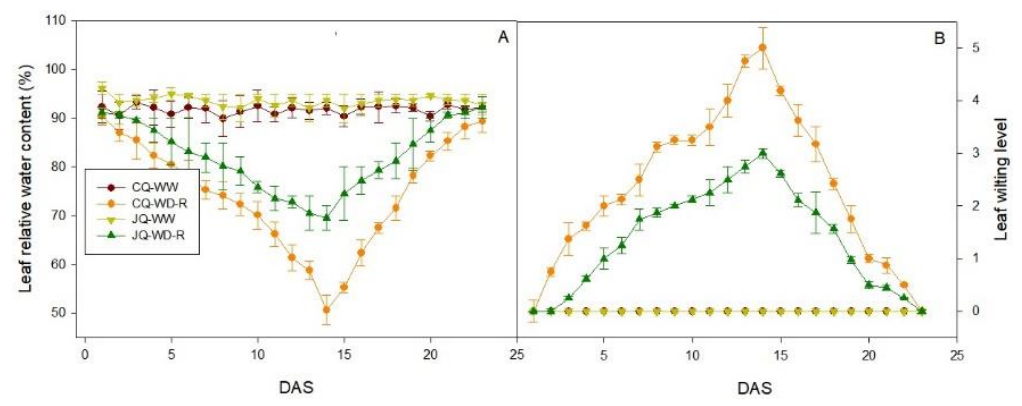

Fig. 1: Effect of drought stress and recovery phase on leaf relative water content $(\mathbf{A})$ and leaf wilting rate $(\mathbf{B})$ of two Tartary buckwheat genotypes

$\mathrm{WW}=$ Well-watered; WD-R= Water deficit to recovery; $\mathrm{CQ}=$ Chanqiao No.1; JQ= JingQiao No.2; DAS= Days after imposition of drought stress

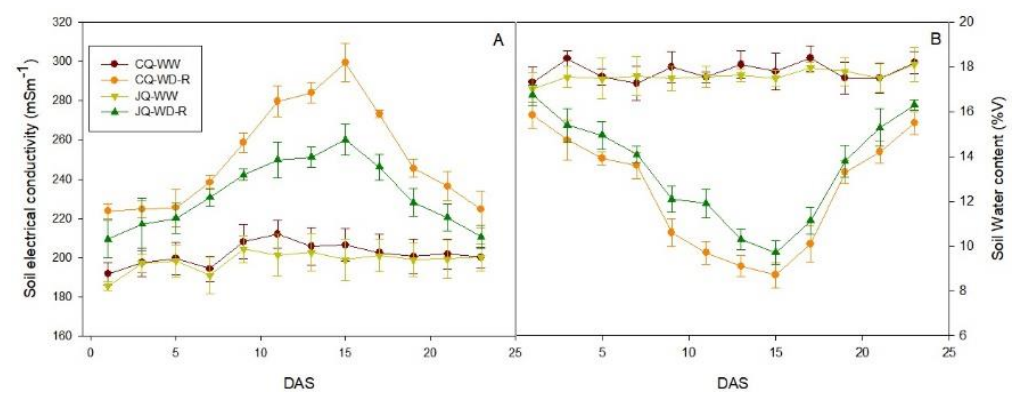

Fig. 2: Effect of drought stress and recovery phase on soil electrical conductivity (A) and soil water content (B) of two Tartary buckwheat genotypes

WW= Well-watered; WD-R= Water deficit to recovery; $\mathrm{CQ}=$ Chanqiao No.1; JQ= JingQiao No.2; DAS= Days after imposition of drought stress

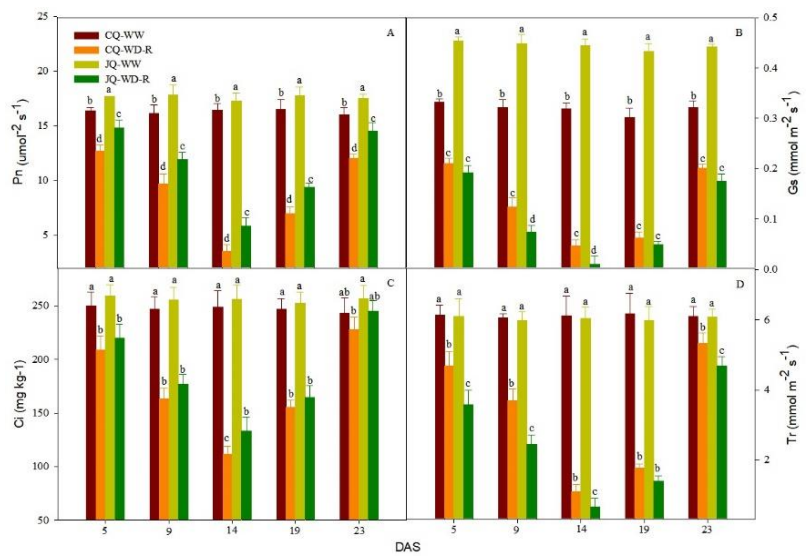

Fig. 3: Effect of drought stress and recovery phase on Pn (A), Gs (B), $\mathrm{Ci}(\mathbf{C})$ and $\operatorname{Tr}(\mathbf{D})$ of two Tartary buckwheat genotypes. Different lowercase letters in each figure indicate significant differences $(P<0.05)$ between treatments on each sampling days $\mathrm{WW}=$ Well-watered; WD-R= Water deficit to recovery; $\mathrm{CQ}=$ Chanqiao No.1; JQ= JingQiao No.2; DAS= Days after imposition of drought stress; Pn=net photosynthetic rate; $\mathrm{Gs}=$ stomatal conductance; $\mathrm{Ci}=$ intercellular $\mathrm{CO} 2$ concentration; $\mathrm{Tr}=$ transpiration rate

deficit in the WD-R treatment in both CQ and JQ plants on days 9 and 14, while Fv/Fm was significantly decreased on day 14. Re-watering induced a decline in $\mathrm{F}_{0}$ and an increase in $\mathrm{Fv} / \mathrm{Fm}$. On days 9 and $14, \mathrm{~F}_{0}$ of CQ under the WD-R treatment was highest among all treatments and genotypes, while Fv/Fm of CQ under WD-R treatment was lowest on day 14.

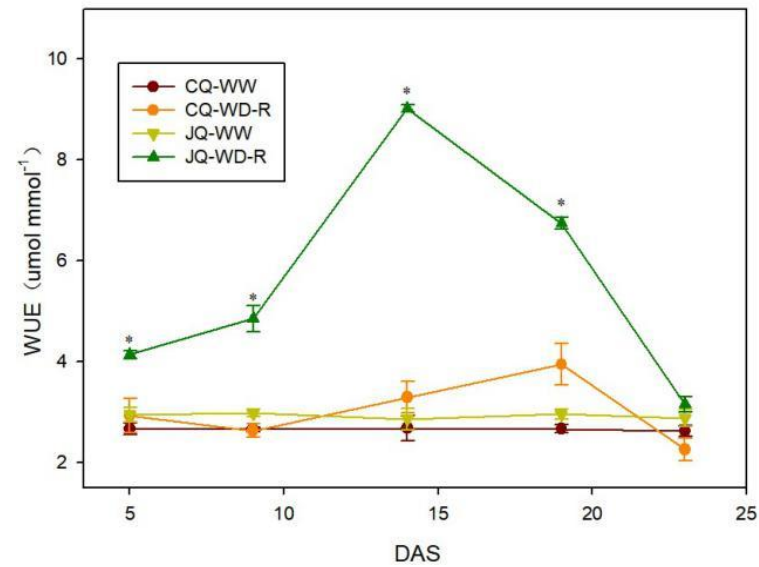

Fig. 4: Effect of drought stress and recovery phase on WUE of two Tartary buckwheat genotypes

WW= Well-watered; WD-R= Water deficit to recovery; $\mathrm{CQ}=$ Chanqiao No.1; JQ= JingQiao No.2; DAS= Days after imposition of drought stress; WUE=water-use efficiency; Asterisks * represent significant differences between treatments on corresponding days $(P<0.05)$

\section{Enzymatic antioxidants}

Across the experiment, MDA and SP contents, and SOD and POD activities in both genotypes under WW treatment were relatively constant (Fig. 6A-D). During the drought phase of the WD-R treatment, MDA content, and SOD and POD activities were significantly enhanced in both 
Water Deficit and Recovery-Induced Changes in Tartary Buckwheat / Intl J Agric Biol, Vol 25, No 2, 2021

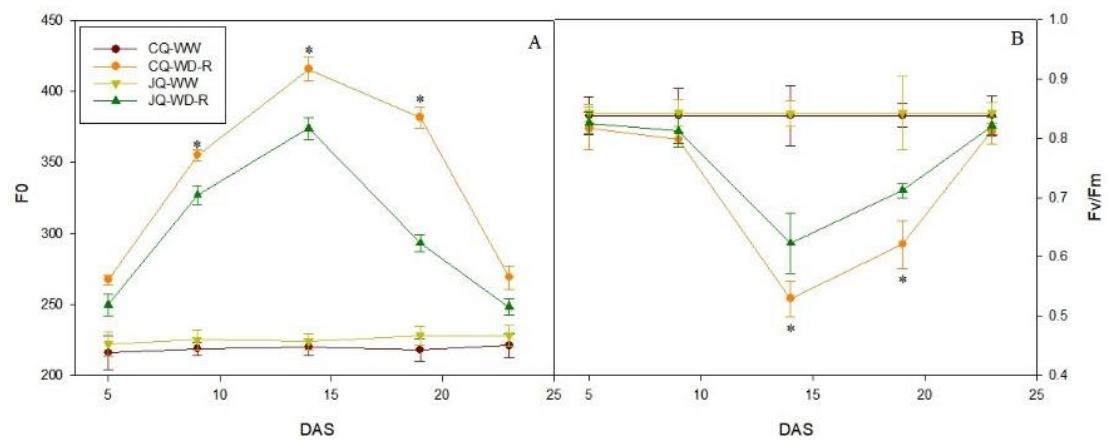

Fig. 5: Effect of drought stress and recovery phase on F0 (A) and Fv/Fm ratio (B) of two Tartary buckwheat genotypes WW= Well-watered; WD-R= Water deficit to recovery; $\mathrm{CQ}=$ Chanqiao No.1; JQ= JingQiao No.2; DAS= Days after imposition of drought stress; F0= initial fluorescence; Fv/Fm ratio= the maximum efficiency of photosystem II (PSII) photochemistry; Asterisks * represent significant differences between treatments on corresponding days $(P<0.05)$

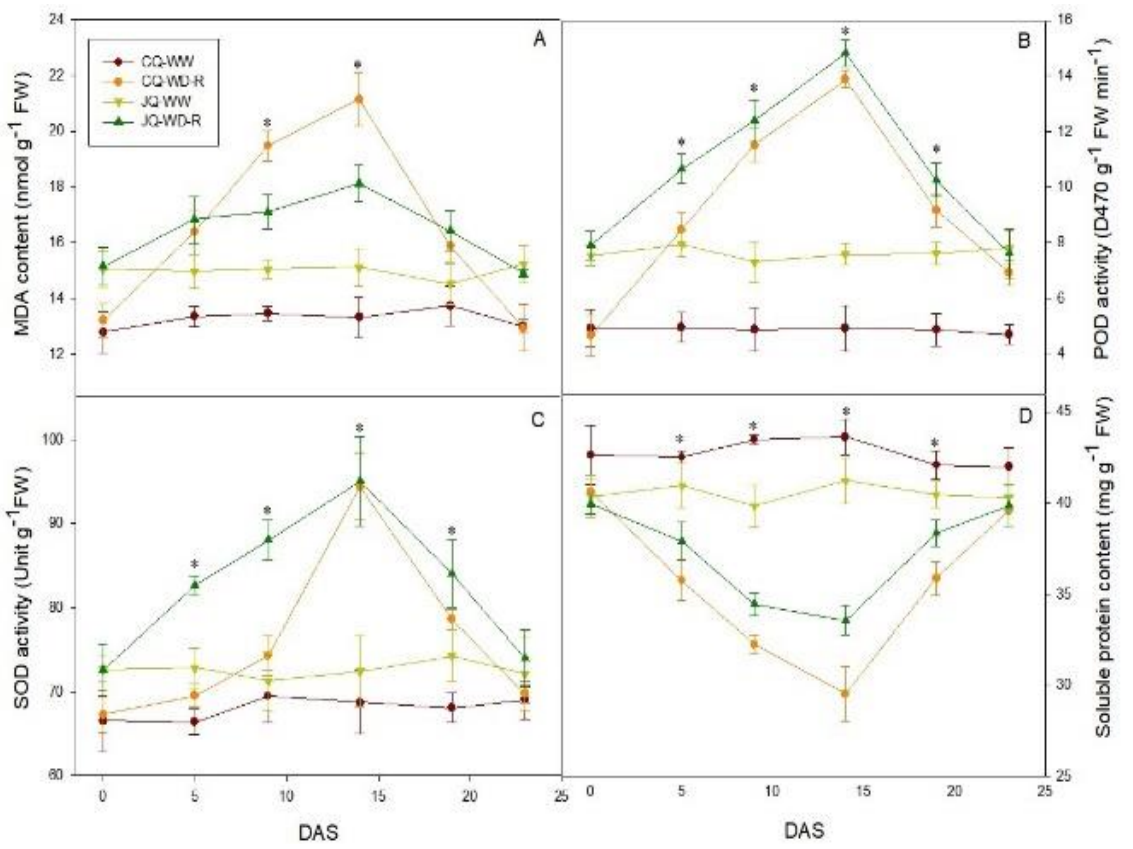

Fig. 6: Effect of drought stress and recovery phase on MDA content (A), POD activity (B), SOD activity (C) and SP content (D) of two Tartary buckwheat genotypes

$\mathrm{WW}=$ Well-watered; WD-R= Water deficit to recovery; $\mathrm{CQ}=$ Chanqiao No.1; JQ= JingQiao No.2; DAS= Days after imposition of drought stress; MDA= Malondialdehyde; $\mathrm{POD}=$ peroxidase; $\mathrm{SOD}=$ superoxide dismutase; $\mathrm{SP}=$ soluble protein; Asterisks * represent significant differences between between treatments on corresponding days $(P<0.05)$

genotypes, and then dropped back to normal levels during the recovery phase. SP showed the opposite trend. MDA content of CQ plants under WD-R treatment was highest out of all treatments on days 9 and 14, leading to a $58.6 \%$ increment compared to CQ plants under the WW treatment on day 14 of the experiment. POD activity in JQ plants under WD-R treatment was highest among all treatments on days 5, 9, 14 and 19. During the drought phase of the WD-R treatment, SOD activity in JQ plants increased steadily, whereas that in CQ plants increased sharply from day 9 to day 14, then decreased back to normal WW levels on day 23. SOD activity in JQ plants under WD-R treatment was highest - significantly higher than that in JQ plants and CQ plants under the WW treatment-on days 5, 9, 14 and 19. The SP content of CQ and JQ plants differed significantly $(P$
$<0.05$ ) between WW and WD-R treatments on days 5, 9, 14 and 19. Among all treatments, SP content was significantly lowest in CQ plants under the WD-R treatment.

\section{Growth, biomass and root-to-shoot ratio}

Plant height, branch number, stem diameter and stem node number for JQ were higher than for CQ under both WW and WD-R conditions (Table 1). The WD-R treatment caused a significant reduction in plant height, branch number, stem diameter and stem node number compared to the WW treatment due to soil dehydration. Among all treatments and genotypes, plant height, stem diameter, branch number and stem node number were significantly lowest for CQ under the WD-R treatment. The same trend 
Table 1: Effect of drought stress and recovery phase on growth, biomass, root to shoot ratio, yield component and yield of two Tartary buckwheat genotypes

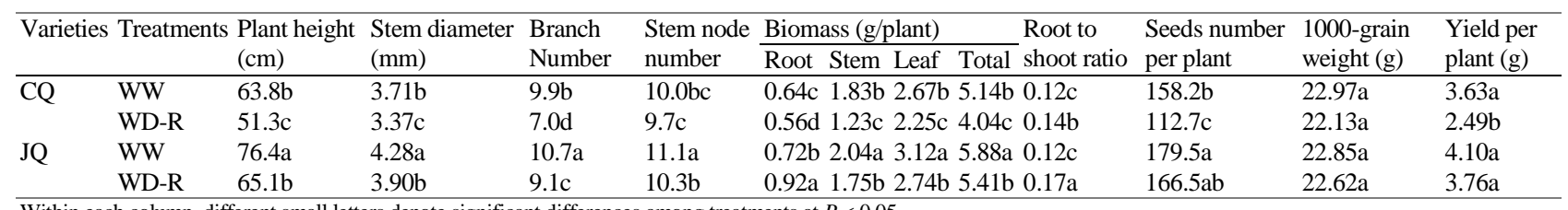

Within each column, different small letters denote significant differences among treatments at $P<0.05$

$\mathrm{WW}=$ Well-watered; WD-R= Water deficit to recovery; $\mathrm{CQ}=$ Chanqiao No.1; JQ= JingQiao No.2

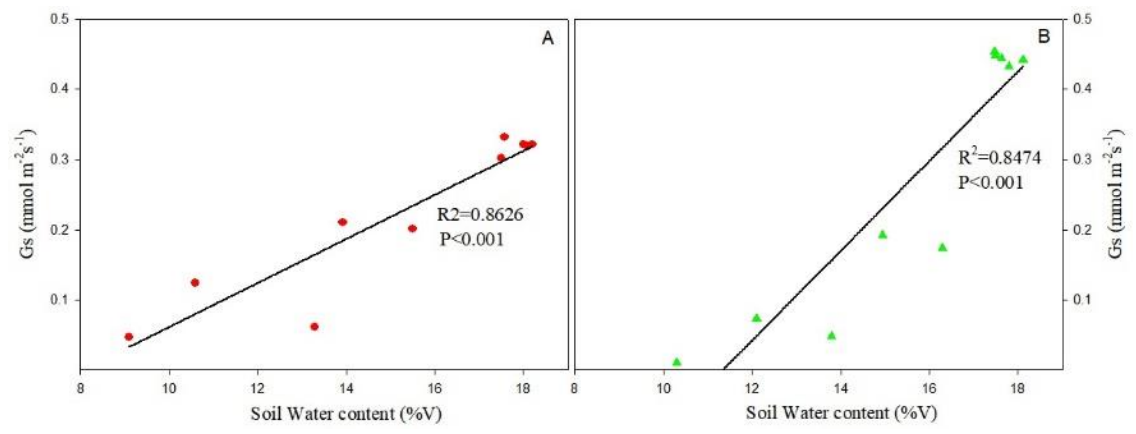

Fig. 7: Relationship between leaf RWC and Gs in CQ (A) and JQ (B) plants during the water stress and recovery phase $\mathrm{CQ}=$ Chanqiao No.1; $\mathrm{JQ}=\mathrm{Jing}$ iao No.2; Gs= stomatal conductance; $\mathrm{RWC}=$ relative water content

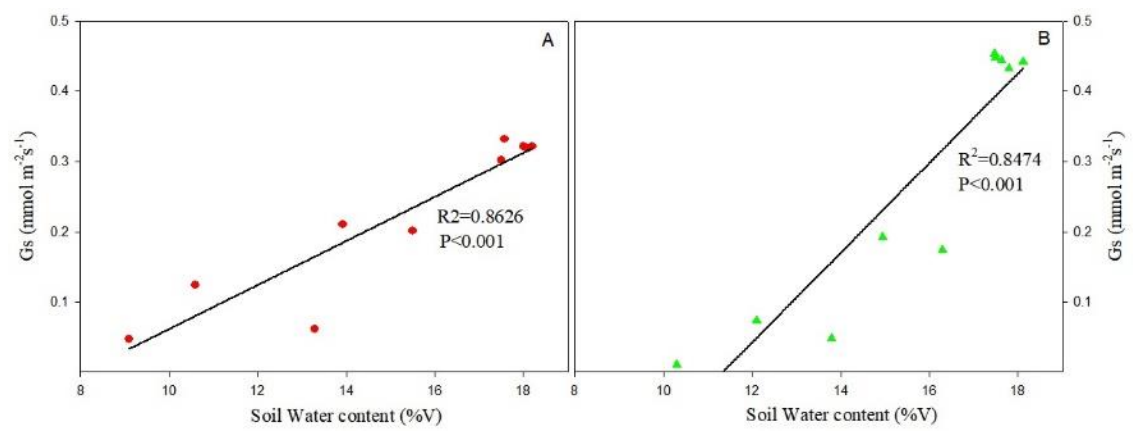

Fig. 8: Relationship between Gs and SWC in CQ (A) and JQ (B) plants during the water stress and recovery phase $\mathrm{CQ}=$ Chanqiao No.1; JQ= JingQiao No.2; Gs= stomatal conductance; SWC= soil water content

was observed for biomass. There were significant differences in biomass (root, stem, leaf and total) between the two genotypes. Root biomass, stem biomass, leaf biomass and total biomass were significant lower under the WD-R treatment than under the WW treatment. Biomass (root, stem, leaf and total) of CQ in the WD-R treatment was the lowest for all water conditions and genotypes. JQ plants had a higher root-to-shoot ratio than CQ plants. Root-to-shoot ratios of CQ and JQ were significantly increased, by 16.7 and $41.7 \%$, respectively, under the WD$\mathrm{R}$ treatment compared to the corresponding WW treatment. Among all treatments, JQ plants under the WD-R treatment had the significantly highest root-to-shoot ratio.

\section{Yield components and total yield}

Under WW conditions, there were no significant differences in seed yield between the two genotypes. However, the seed yield of CQ decreased more than that of JQ under the WD$\mathrm{R}$ treatment (Table 1). The yield of CQ reached under the WD-R treatment was $31.4 \%$ of that under to the WW treatment, but there was no significant yield loss for JQ grown under the WW vs. WD-R treatments. Seed number per JQ plant was significantly higher than that per CQ plant under both WW and WD-R conditions. Seed number per CQ and JQ plant decreased under the drought part of the WD-R treatment. The number of seeds per CQ and JQ plant decreased significantly, by 28.8 and $12.8 \%$, respectively, under the WD-R treatment compared to the corresponding WW treatment. There were no significant differences in grain weight among genotypes or treatments.

\section{Correlation analysis}

Relationships between Gs, leaf RWC and SWC were studied to determine those that might indicate responses to 
drought stress and recovery in CQ and JQ plants. The Gs was positively correlated with leaf RWC in CQ and JQ plants $(P<0.01$; Fig. 7). Positive correlations were also found in $\mathrm{CQ}\left(\mathrm{R}^{2}=0.8626, P<0.001\right.$; Fig. $\left.8 \mathrm{~A}\right)$ and JQ $\left(\mathrm{R}^{2}=\right.$ $0.8474, P<0.001$; Fig. 8 B) plants between SWC and Gs.

\section{Discussion}

Water loss, photosynthetic inhibition, growth restriction, and cell membrane damage are the most common symptoms in plants under drought stress (Farooq et al. 2009; Bodner et al. 2015; Hussain et al. 2017). Some of these effects can be restrictive or even devastating. In this study, the CQ and JQ plants survived 14 days of progressive water deficit. A significant difference in LW was found between WW and WD-R treatments. Compared to JQ plants, CQ plants were more sensitive to dehydration, as wilting appeared earlier and were more severe. The leaf is an important organ for photosynthesis. Under normal conditions, with sufficient water for photosynthesis, plants utilize a large fragment of light constant. When subjected to drought, the equilibrium between light capture and water utilization can be disrupted (Chaves et al. 2003). At first, CQ and JQ plants showed higher WUE to cope with decreasing water availability. To minimize water loss and maintain adequate leaf water status during dehydration in the WD-R treatment, CQ and JQ plants initially reduced Gs to adapt to the drought, along with a significant reduction in $\operatorname{Tr}$ (Chaves et al. 2002; Pompelli et al. 2010; Tardieu 2012). Furthermore, the dramatic reduction in $\mathrm{Pn}, \mathrm{Gs}$ and $\mathrm{Ci}$ suggested that photosynthesis is mainly restricted by stomatal limitations in the two Tartary buckwheat genotypes (Flexas and Medrano 2002; Hu et al. 2018). A decline in leaf RWC is another way for plants to increase water availability, contributing to increased carbon delivery to the roots (Rosales-Serna et al. 2004). As expected, a large decrease in leaf RWC was found for both genotypes under the WD-R treatment. In addition, correlation analysis suggested that the two plants lost part of their leaf RWC at low stomatal aperture. At the same time, they reduced Gs to cope with the decreasing SWC. A similar finding was reported in Jatropha curcas (Jatropha curcas L.) (Sapeta et al. 2013). Compared to the CQ plants, JQ plants exhibited higher WUE by lowering transpiration through a decrease in Gs, supporting JQ as a drought-tolerant genotype (Blum 2009; Rahbarian et al. 2011). Upon re-watering, the stomata reopened and leaf RWC recovered rapidly, with increasing Pn. Comparatively higher Pn of WD-R-treated JQ plants was also detected after re-watering, implying better photosynthetic recovery from water deficit in the JQ plants.

On the other hand, drought usually causes photoinhibition when light exceeds the capacity for photosynthesis (Shi et al. 2016). The two Tartary buckwheat plants had to subtract excrescent light by preventing (Havaux and Tardy 1999) or dissipating (Chaves et al. 2003) absorbance. For the WD-R-treated plants, increased LW on days 5, 9 and 14 was an effective way to minimize light absorption. At the same time, a decrease in Fv/Fm was observed in the two genotypes during the drought phase of the WD-R treatment. Fv/Fm is an important indicator of PSII photochemical efficiency. Thus, plants exhibited a reduction in PSII photochemical efficiency (Rahbarian et al. 2011), together with yield losses, under drought conditions (Pathan et al. 2014). In the current study, decreased Fv/Fm occurred after net assimilation decreased, reinforcing the notion that stomatal limitations primarily limit Pn, rather than photochemistry (Sapeta et al. 2013). PSII efficiency was limited due to reduced $\mathrm{CO}_{2}$ supply, which has a negative effect on the Calvin cycle (Sapeta et al. 2013). JQ plants maintained a higher Fv/Fm ratio compared to CQ plants during the drought, suggesting rapid adjustments in the former to avoid the decline in PSII photochemical efficiency. Upon re-watering, Fv/Fm quickly recovered to pre-drought levels, suggesting that the damage had been successfully repaired.

Reactive oxygen species (ROS) are frequently generated under drought stress, causing serious oxidative damage to plants (Oliver et al. 2010). Antioxidant enzymes function in quenching the ROS (Luna et al. 2005). Of these, SOD, the first key enzyme in the active oxygen-scavenging system, plays a crucial role in catalyzing superoxide free radical dismutation into $\mathrm{H}_{2} \mathrm{O}_{2}$ and $\mathrm{O}_{2}$ (Blokhina et al. 2003). SOD activity increased in the leaves of the two Tartary buckwheat genotypes under water stress. The same trend was found for leaf POD activity at the end of the drought stress. Increased activities of SOD, POD, and catalase in response to water deficit have been reported in potato (Solanum tuberosum L.) (Boguszewska et al. 2010). Drought-tolerant white clover (Trifolium repens L.) retained significantly higher POD and SOD activity in roots and leaves during drought treatments (Li et al. 2012). We also found that JQ leaves maintained higher SOD and POD activity on days 5 and 9 of the drought phase compared to CQ leaves. The steadily decreasing trend of SOD and POD activity during the recovery phase revealed the successful reinstatement of osmosis and antioxidation. Typically, ROSinduced oxidative damage under water stress can be adjusted by osmoregulatory molecules, such as proline, soluble sugars, SP, flavonoids, and late-embryogenesis abundant proteins (Reddy et al. 2004; Claeys and Inze 2013). These substances help the membranes defend against drought stress and stimulate plants to absorb more water to retain normal metabolic functions (Claeys and Inze 2013). The MDA, a marker of oxidative stress stemming from lipid peroxidation, is generally employed to assay oxidative damage (Sarker and Oba 2018). In the present study, MDA content in the leaves of both Tartary buckwheat genotypes increased during the drought phase, but a slower increase in MDA was found in the JQ vs. CQ plants, which could be attributed to the former's better tolerance to drought stress.

Along with the physiological responses to water stress, morphological adaptions were observed. The most prominent of these in the two Tartary buckwheat genotypes 
were the reductions in plant height, stem node number and branch number in the water-stressed plants. Genotypes with maximum root length and minimum shoot length under water deficit are regarded as drought-tolerant (Chaves et al. 2003; Oliver et al. 2010). A significant increase in root-toshoot ratio was found in WD-R plants compared to WW plants, greater in JQ vs. CQ plants. Such responses might be attributed to root system development: root growth in JQ was relatively more enhanced than in CQ during the drought phase.

Drought-induced yield loss is a major problem in many plants (Bodner et al. 2015). The primary yielddetermining components-grain number and grain weight - are influenced by water stress, depending on the duration and magnitude of the drought, and the phenological stage at which it occurs (Farooq et al. 2014; Bodner et al. 2015). In the present study, water deficit at the flowering stage markedly reduced seed number in CQ plants. Yield loss due to lack of water in CQ plants reached $31.4 \%$, mainly due to the reduced number of seeds, whereas neither yield nor seed number were significantly affected by the water deficit in JQ plants. Thus, the higher yield and higher number of seeds in the JQ plants might have been a result of the higher photosynthetic activity with more functional leaves and lower transpiration, due to increased WUE, in those plants under water stress.

\section{Conclusion}

Findings of this study imply that physiological acclimation of Tartary buckwheat variety JQ to progressive drought includes elevated water-use efficiency through stomatal closure, and decreased photosynthesis rate, transpiration rate and PSII photochemical efficiency. At the same time, JQ has a more active ROS-scavenging system with higher activities of superoxide dismutase and peroxidase, which may be one of the crucial routes to avoiding oxidative membrane damage and lipid peroxidation. Furthermore, accumulation of malondialdehyde helped maintain cell turgor and metabolic functions. Upon re-watering, these parameters eventually returned to, or close to, normal levels. These combined strategies enable JQ plants to survive without significant yield loss under drought conditions with unreliable precipitation.

\section{Acknowledgements}

All authors acknowledge the financial grant from the Key Research and Development Program of the Ministry of Science and Technology of China (2019YFD1001302/ 2019YFD1001300), National Natural Science Foundation of China (31601260), the Applied Basic Research Programs of the Project Science and Technology Commission of Sichuan Province (2016JY0209) and Supported by the earmarked fund for China Agriculture Research System (CARS-08-02A).

\section{Author Contributions}

Y.W and D.X analyzed the data, wrote the manuscript and revised the manuscript; J.O, X.G and L.L conducted the experiment and collected data; X.W, Q.W, L.Z and G.Z reviewed and supervised the manuscript; all authors mentioned approved the final manuscript.

\section{References}

Barrs H, P Weatherley (1962). A re-examination of the relative turgidity technique for estimating water deficits in leaves. Aust J Biol Sci 15:413-428

Blokhina O, E Virolainen, K Fagerstedt (2003). Antioxidants, oxidative damage and oxygen deprivation stress: A review. Ann Bot 91:179-194

Blum A (2009). Effective use of water (EUW) and not water-use efficiency (WUE) is the target of crop yield improvement under drought stress. Field Crops Res 112:119-123

Bodner G, A Nakhforoosh, HP Kaul (2015). Management of crop water under drought: A review. Agron Sustain Dev 35:401-442

Boguszewska D, M Grudkowska, B Zagdan'Ska, ME ÇAlışKan, PC Struik (2010). Drought-responsive antioxidant enzymes in potato (Solanum tuberosum L.). Potato Res 53:373-382

Bradford M (1976). A rapid and sensitive method for the quantitation of microgram quantities of protein utilizing the principle of protein-dye binding. Anal Biochem 72:248-254

Chaves MM, JP Maroco, JS Pereira (2003). Understanding plant responses to drought-from genes to the whole plant. Funct Plant Biol 30:239-264

Chaves MM, JS Pereira , J Maroco, M Rodrigues, CP Ricardo, ML Osorio, I Carvalho, T Faria, C Pinheiro (2002). How plants cope with water stress in the field. Photosynthesis and growth. Ann Bot 89:907-916

Chimungu JG, KM Brown, JP Lynch (2014). Large root cortical cell size improves drought tolerance in maize. Plant Physiol 166:2166-2178

Claeys H, D Inze (2013). The agony of choice: How plants balance growth and survival under water-limiting conditions. Plant Physiol 162:1768-1779

Delpérée C, J Kinet, S Lutts (2003). Low irradiance modifies the effect of water stress on survival and growth-related parameters during the early developmental stages of buckwheat (Fagopyrum esculentum). Physiol Plantarum 119:211-220

Dhindsa RS, PD Pamela, AT Thorpe (1981). Leaf senescence: Correlated with increased levels of membrane permeability and lipid peroxidation, and decreased levels of superoxide dismutase and catalase. J Exp Bot 32:93-101

Dias MC, W Bruggemann (2010). Limitations of photosynthesis in Phaseolus vulgaris under drought stress: Gas exchange, chlorophyll fluorescence and Calvin cycle enzymes. Photosynthetica 48:96-102

Farooq M, A Wahid, N Kobayash, D Fujita, SMA Basra (2009). Plant drought stress: Effects, mechanisms and management. Agron Sustain Dev 29:185-212

Farooq M, F Nadeem, MY Arafat, M Nabeel, S Musadaq, SA Cheema, A Nawaz (2018) Exogenous application of allelopathic water extracts helps improving tolerance against terminal heat and drought stresses in bread wheat (Triticum aestivum L. Em. Thell.). J Agron Crop Sci 204:298-312

Farooq M, M Hussain, KHM Siddique (2014) Drought stress in wheat during flowering and grain-filling periods. Crit Rev Plant Sci 33:331-349

Farooq M, M Hussain, S Ul-Allah, KHM Siddique (2019). Physiological and agronomic approaches for improving water-use efficiency in crop plants. Agric Water Manage 219:95-108

Farooq M, M Rizwan, A Nawaz, A Rehman, R Ahmad (2017) Application of natural plant extracts improves the tolerance against combined terminal heat and drought stresses in bread wheat. J Agron Crop Sci 203:528-538 
Flexas J, H Medrano (2002). Drought-inhibition of photosynthesis in $\mathrm{C}_{3}$ plants: Stomatal and non-stomatal limitations revisited. Ann Bot 89:183-189

Franks PJ, TW Doheny-Adams, ZJ Britton-Harper, JE Gray (2015). Increasing water-use efficiency directly through genetic manipulation of stomatal density. New Phytol 207:188-195

Giannopolitis CN, SK Ries (1977). Superoxide dismutases, 1: Occurrence in higher plants [Corn, oats, peas]. Plant Physiol 59:309-314

Havaux M, F Tardy (1999). Loss of chlorophyll with limited reduction of photosynthesis as an adaptive response of Syrian barley landraces to high-light and heat stress. Funct Plant Biol 6:569-578

Hu W, SB Tian, Q Di, SH Duan, K Dai (2018). Effects of exogenous calcium on mesophyll cell ultrastructure, gas exchange, and photosystem II in tobacco (Nicotiana tabacum Linn.) under drought stress. Photosynthetica 56:1204-1211

Hussain M, S Farooq, W Hasan, S Ul-Allah, M Tanveer, M Farooq, A Nawaz (2018). Drought stress in sunflower: Physiological effects and its management through breeding and agronomic alternatives. Agric Water Manage 201:152-167

Hussain M, M Farooq, DJ Lee (2017). Evaluating the role of seed priming in improving drought tolerance of pigmented and non-pigmented rice. J Agron Crop Sci 203:269-276

Li Z, Y Peng, X Ma (2012). Different response on drought tolerance and post-drought recovery between the small-leafed and the large-leafed white clover (Trifolium repens L.) associated with antioxidative enzyme protection and lignin metabolism. Acta Physiol Plantarum 35:213-222

Luna CM, GM Pastori, S Driscoll, G Karin, B Stephanie (2005). Drought controls on $\mathrm{H}_{2} \mathrm{O}_{2}$ accumulation, catalase (CAT) activity and CAT gene expression in wheat. $J$ Exp Bot 56:417-423

Nakono Y, K Asoda (1980). Hydrogen peroxide is scavenged by ascorbatespecific peroxidase in spinach chloroplasts. Plant Cell Physiol 22:867-880

Nguyen KH, C Ha Van, R Nishiyama, Y Watanabe, MA LeyvaGonzalez, Y Fujita, UT Tran, WQ Li, M Tanaka, M Seki, GE Schaller, L Herrera-Estrella, LSP Tran (2016). Arabidopsis type B cytokinin response regulators ARR1, ARR10, and ARR12 negatively regulate plant responses to drought. Proc Natl Acad USA 113:3090-3095

Oliver M, J Cushman, K Koster (2010). Dehydration tolerance in plants. Meth Mol Biol 639:3-24

Pathan SM, JD Lee, DA Sleper, FB Fritschi, RE Sharp, TE Carter, RL Nelson, CA King, WT Schapaugh, MR Ellersieck, HT Nguyen, JG Shannon (2014). Two soybean plant introductions display slow leaf wilting and reduced yield loss under drought. J Agron Crop Sci 200:231-236

Pompelli MF, R Barata-Luis, HS Vitorino, ER Goncalves, EV Rolim, MG Santos, JS Almeida-Cortez, VM Ferreira, EE Lemos, L Endres (2010). Photosynthesis, photoprotection and antioxidant activity of purging nut under drought deficit and recovery. Biomass Bioener $34: 1207-1215$
Rahbarian R, R Khavari-Nejad, A Ganjeali, A Bagheri, F Najafi (2011). Drought stress effects on photosynthesis, chlorophyll fluorescence and water relations in tolerant and susceptible chickpea (Cicer Arietinum L.) genotypes. Acta Biol Cracov Bot 53:47-56

Reddy AR, KV Chaitanya, M Vivekanandan (2004). Drought-induced responses of photosynthesis and antioxidant metabolism in higher plants. J Plant Physiol 161:1189-1202

Rosales-Serna R, J Kohashi-Shibata, JA Acosta-Gallegos, C Trejo-López, J Ortiz-Cereceres, JD Kelly (2004). Biomass distribution, maturity acceleration and yield in drought-stressed common bean cultivars. Field Crops Res 85:203-211

Sapeta H, JM Costa, T Lourenco, J Maroco, LV der, MM Oliveira (2013). Drought stress response in Jatropha curcas: Growth and physiology. Environ Exp Bot 85:76-84

Sarker U, S Oba (2018). Drought stress effects on growth, ros markers, compatible solutes, phenolics, flavonoids, and antioxidant activity in Amaranthus tricolor. Appl Biochem Biotechnol 186:999-1016

Shi Q, YL Yin, ZQ Wang, WC Fan, JF Hua (2016). Physiological acclimation of taxodium hybrid 'Zzhongshanshan 118' plants to short-term drought stress and recovery. Hortscience 51:1159-1166

Simmons AR, DC Bergmann (2016). Transcriptional control of cell fate in the stomatal lineage. Curr Opin Plant Biol 29:1-8

Tardieu F (2012). Any trait or trait-related allele can confer drought tolerance: Just design the right drought scenario. J Exp Bot 63:25-31

Vasquez-Robinet C, SP Mane, AV Ulanov, JI Watkinson, VK Stromberg, DD Koeyer, R Schafleitner, DB Willmot, M Bonierbale, HJ Bohner, R Grene (2008). Physiological and molecular adaptations to drought in Andean potato genotypes. J Exp Bot 59:2109-2123

Wu Y, DJ Cosgrove (2000). Adaptation of roots to low water potentials by changes in cell wall extensibility and cell wall proteins. $J$ Exp Bot $51: 1543-1553$

Xiang DB, W Wei, JY Ouyang, LQ Le, G Zhao, LX Peng, Y Wan (2020). Nitrogen alleviates seedling stage drought stress response on growth and yield of Tartary Buckwheat. Intl J Agric Biol 24:1167-1177

Yoo CY, HE Pence, JB Jin, K Miura, MJ Gosney, PM Hasegawa, MV Mickelbart (2010). The Arabidopsis GTL1 transcription factor regulates water use efficiency and drought tolerance by modulating stomatal density via transrepression of SDD1. Plant Cell $22: 4128-4141$

Yoshida T, J Mogami, K Yamaguchi-Shinozaki (2014). ABA-dependent and $\mathrm{ABA}$-independent signaling in response to osmotic stress in plants. Curr Opin Plant Biol 21:133-139

Zhang L, X Li, B Ma, Q Gao, H Du, Y Han, Y Li, Y Cao, M Qi, Y Zhu (2017). The Tartary Buckwheat genome provides insights into rutin biosynthesis and abiotic stress tolerance. Mol Plant 10:1224-1237

Zhou ML, CL Wang, LP Qi, XB Yang, ZM Sun, Y Tang, YX Tang, JR Shao, Y Wu (2015). Ectopic expression of Fagopyrum tataricum FtMYB12 improves cold tolerance in Arabidopsis thaliana. J Plant Growth Regul 34:362-371

Zhu F (2016). Chemical composition and health effects of Tartary buckwheat. Food Chem 203:231-245 